\title{
DESIGN OF 2.4 GHZ TRANSCEIVER FOR WIRELESS COMMUNICATION
}

\author{
Muhanned AL-RAWI \\ University of Ibb, Yemen \\ muhrawi@yahoo.com
}

\begin{abstract}
The receiver and the transmitter are usually a cascade of several circuits. This paper presents a study of each of the individual components that comprise both the transmitter and the receiver. The design tasks for each component are described as well as the factors to be put into consideration. The simulations of the various components are done using microwave office and Proteus 7 professional. The results obtained for the receiver are satisfactory and fully demonstrated the functioning of the receiver.
\end{abstract}

KEYWORDS: $2.4 \mathrm{GHz}$ transceiver, wireless communication, design

\section{Introduction}

A transceiver is a tool which has both a transmitter and a receiver, that are mixed and share commonplace circuitry or a single housing. If no circuitry is common between the transmitter and the receiver features, the tool is a transmitter-receiver. The transmitter and receiver function concurrently in a full duplex device however can run in various time slots in system of half duplex. The transmitter must rent narrowband modulation, an amplification and filtering process to keep away from leakage to adjoining channels. The receiver should be able to receive a weak radio frequency signal, amplify it sufficiently and convert it into a baseband signal while sufficiently rejecting strong neighboring interferers (Camargo \& Topacio, 1997).

There are various architectures that are used in the design of transceivers. The most commonly used include the heterodyne architecture, the direct conversion architecture and the low intermediate frequency (IF) architecture (Rahayu, Din \& Abdulrahman, 2003). Of the three, the most popularly used is the heterodyne architecture since it offers the best performance compared to the other architectures (Debski, Winkler, Genschow $\&$ Kraemeret, 2011). This is the architecture that has been used for the receiver design in this paper. Due to the fact that most devices used in the design of the transceiver exhibit non-linear behavior, some important factors to consider in the design of the transceiver are highlighted. These factors include: noise, harmonics, intermodulation distortion, image problem and the half IF problem. These factors determine the fidelity of the signal as it passes through the transmitter or the receiver therefore if they are not put into consideration the signal will be highly distorted (Basavaraj, 2018). Since the $2.4 \mathrm{GHz}$ frequency range falls within the microwave frequency range, the S-parameters are considered. This is because at microwave frequencies the analysis is more involving than in ordinary circuit theory since propagation effects have to be put into consideration owing to very short wavelengths. Each of the components that make up the transmitter 
and receiver are covered in detail with their functions and requirements clearly stated. The circuit diagrams are also given. The simulations are done for the individual sub-circuits or components that constitute the transceiver and the simulations for the entire transmitter and the receiver paths are also documented.

\section{Transceiver Architecture}

\subsection{Receiver}

In general, the receiver is described from the gate connected to the antenna of receiver to the device of analogue to digital converter (ADC). There are several receiver architectures in use, but in this paper, heterodyne architecture is considered.

\subsubsection{Heterodyne Architecture}

This architecture is the most famous one utilized in communication transceivers system. It normally consists of three sections, the radio frequency (RF), IF and base-band (BB) sections. It is based totally on the heterodyne procedure of mixing the incoming signal with offset frequency local oscillator (LO) in a nonlinear tool to produce an IF signal in the receiver or to generate RF signal from its IF model inside the transmitter. The nonlinear tool which executes the heterodyne system is known as a frequency mixer or frequency converter.
In this design, the process of frequency translation can be achieved more than once and as a consequence it is able to have more than one intermediate frequencies and more than one IF blocks. The intermediate frequency may be produced with the aid of an incoming signal with a frequency both above or below the local oscillator. Between those frequencies, the one corresponding to the unwanted signal is referred to as frequency of image. To avoid the possible image interfering with the favored signal, enough filtering is carried out earlier than frequency changing. The process of channel filtering of a heterodyne receiver is executed in the IF block via passive filters with high selectivity. Most of the favored signal amplification is provided with the aid of the IF blocks. This architecture has the high-quality performance while as compared with the others for this reason it is far the most popularly utilized structure in wireless communication systems. The primary disadvantages of this structure are its high price and large electrical power consumption. A large fraction of the price goes into IF and RF filters. The block diagram of the heterodyne receiver is shown in Figure no. 1. As shown in Figure no. 1, heterodyne receiver consists of antenna, Band-pass Filter (BPF), Low Noise Amplifier (LNA), Mixer, IF filter, and IF amplifier.

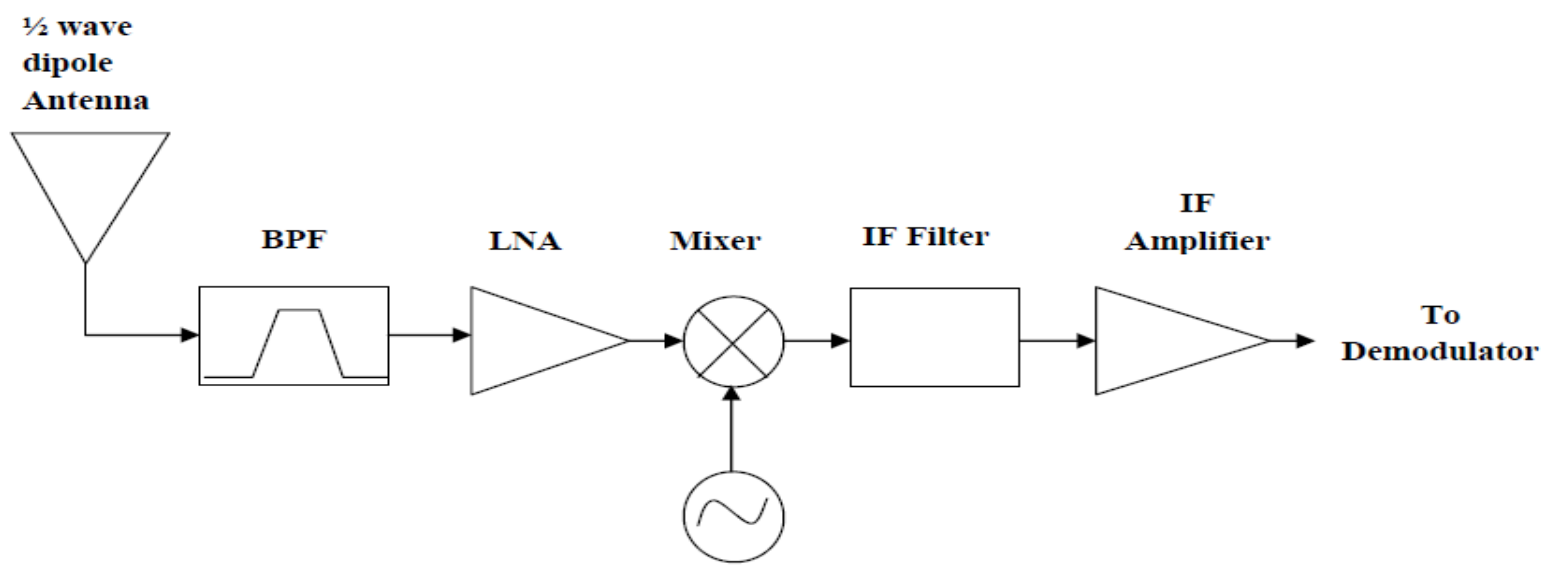

Local Oscillator

Figure no. 1: Heterodyne receiver Block diagram 


\subsubsection{Receiver Requirements}

Some very important receiver requirements include:

1. High gain: This is necessary to repair the low electricity of the received signal to a degree near its original baseband signal.

2. Selectivity: Good selectivity ensures that the receiver receives the desired signal while rejecting the undesired frequencies such as image frequencies and interference from other channels.

3. Isolation: There should be good isolation between the receiver and the transmitter to avoid saturation of the receiver.

4. Sensitivity: This is described as the minimum signal a device can discover with desirable signal to noise ratio. The sensitivity of the receiver may be computed from the noise figure (NF) of the receiver.

\subsection{Transmitter}

An RF transmitter is described from a digital to analogue converter (DAC) to the gate connected to the antenna of the transmitter. The transmitter basically consists of the following:

1. IF filter;

2. Mixer;

3. Local oscillator;

4. RF Band-pass filter;

5. Power amplifier(PA);

6. Antenna.

An RF transmitter achieves upconversion and power gain. In assessment to the sort of procedures invented for RF receivers, transmitter structures are located in only a few forms. This is due to the fact issues consisting of noise, interference rejection, and band selectivity are greater at ease in transmitters than in receivers. The block diagram of the transmitter is shown in Figure no. 2.

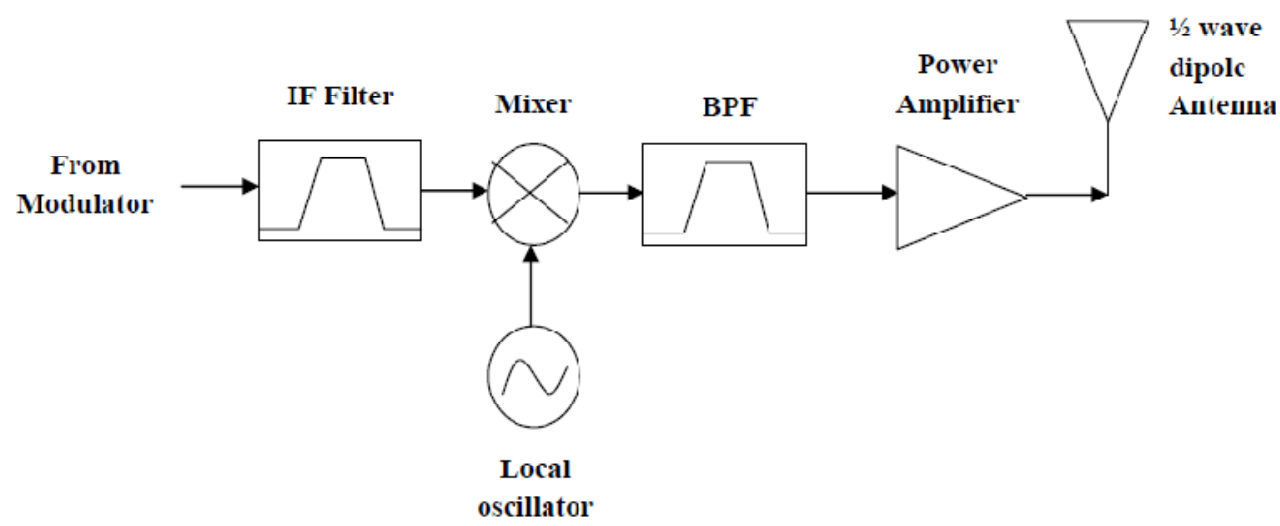

Figure no. 2: Block diagram of a transmitter

The vital parameters of a transmitter include output power and the fidelity of the transmission waveform. This is computed via modulation accuracy or quality factor of the waveform. Undesired emissions along with adjacent channel electricity and inband and out-of band noise have to be well described in specifications of cellular mobile transmitter. That is because the undesirable emissions may additionally jam other wireless structures. Nonlinearity of the transmitter that specifically comes from the PA consequences in an growth of adjacent channel strength emissions. Two transmitter structures are taken into account. Those are: direct conversion transmitters and two-step transmitter.

\subsubsection{Direct Conversion Transmitter}

In this structure, the transmitted carrier frequency is equal to the frequency of the local oscillator inside the baseband level. 
The modulator is directly followed without delay by the PA then followed by a matching network. The characteristic of the matching network is to give maximum power transfer to the antenna and to filter out-of-band additives that result from the nonlinearities of the PA. This is depicted in Figure no. 3. Since the baseband signal is generated inside the transmitter, it is adequately strong, therefore the noise inside the transmitter mixer is much less vital than in the receiver mixer.

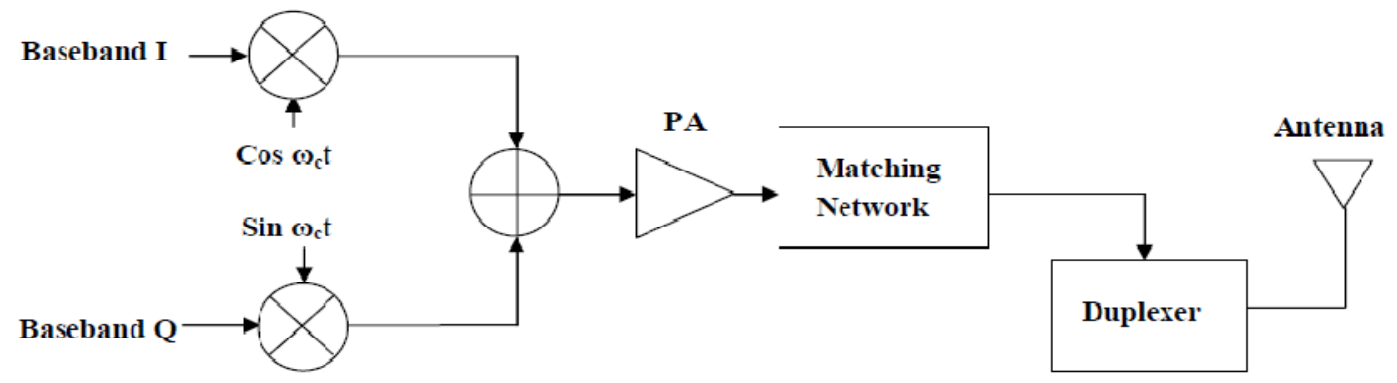

Figure no. 3: Direct conversion transmitter

This architecture however is suffering from annoying of the local oscillator with the aid of the PA. This problem is arising because of the truth that the PA output is a modulated signal with high energy and a spectrum targeted around the local oscillator frequency. The PA is corrupting the oscillator spectrum via a mechanism known as injection pulling or injection locking. This impact is worsened if the PA is tuned OFF and ON periodically to keep on power. This impact can be alleviated if the PA output spectrum is satisfactorily better or lower than the oscillator frequency. This can be done by adding or subtracting the output frequency of another oscillator. This has been taken care of by the two-step transmitter architecture.

\subsubsection{Two-Step Transmitter}

In this architecture, the baseband I and Q channels undergo quadrature modulation at a lower frequency referred to as the IF. The signal is then passed through an IF band-pass filter to suppress the harmonics of the IF signal, after which it is passed through an up-converting mixer. It is then passed through an RF band-pass filter which removes the unwanted sidebands centered around $\omega_{L O}-\omega_{I F}$ and finally through the PA and to the antenna. Figure no. 4 shows the two-step transmitter. This architecture has the following advantages:

1. Since the quadrature modulation is achieved at much lower frequencies, the I and Q matching is superior leading to less cross-talk.

2. Since the LO frequency is lower than the frequency of waveform at the output of the PA, this architecture does not suffer from injection pulling.

A difficulty of this architecture is that the RF bandpass filter should refuse the undesired sideband by a factor of about $50 \mathrm{~dB}$ to $60 \mathrm{~dB}$.

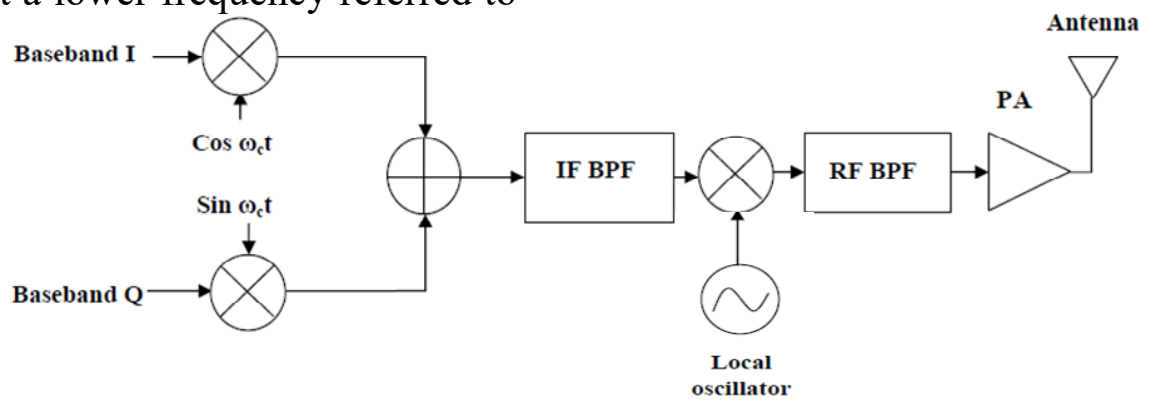

Figure no. 4: Two-step transmitter 


\section{Simulation Results}

\subsection{RF Bandpass Filter}

The simulation result of BPF is as shown in Figure no. 5. It is seen that the filter is designed to have a $3 \mathrm{~dB}$ pass-band of $90 \mathrm{MHz}$. It is designed to have a return loss of $2 \mathrm{~dB}$, an attenuation of $60 \mathrm{~dB}$ at the frequencies $2.28 \mathrm{GHz}$ and $2.62 \mathrm{GHz}$. The ripple is $3 \mathrm{~dB}$. The choice of filter parameters is dependent on the out-of-band rejection and the in-band noise. However, since the signal received by the antenna is usually of very low power, the in-band noise is given the greater consideration to ensure minimum noise possible. Moreover, being the first stage after the antenna, it should have minimal noise since a lossy circuit "Magnifies" the noise figure in the succeeding stage by the attenuation factor.

From the simulation result, it is also seen that the input reflection coefficient $\left(\mathrm{S}_{11}\right)$ is minimum around the center frequency of the filter. This is a desirable characteristic since it ensures that there is maximum power transmission within the frequency band of interest while minimum power transmission is achieved outside the frequency band of interest. $S_{21}$ is the transmission coefficient of the filter.

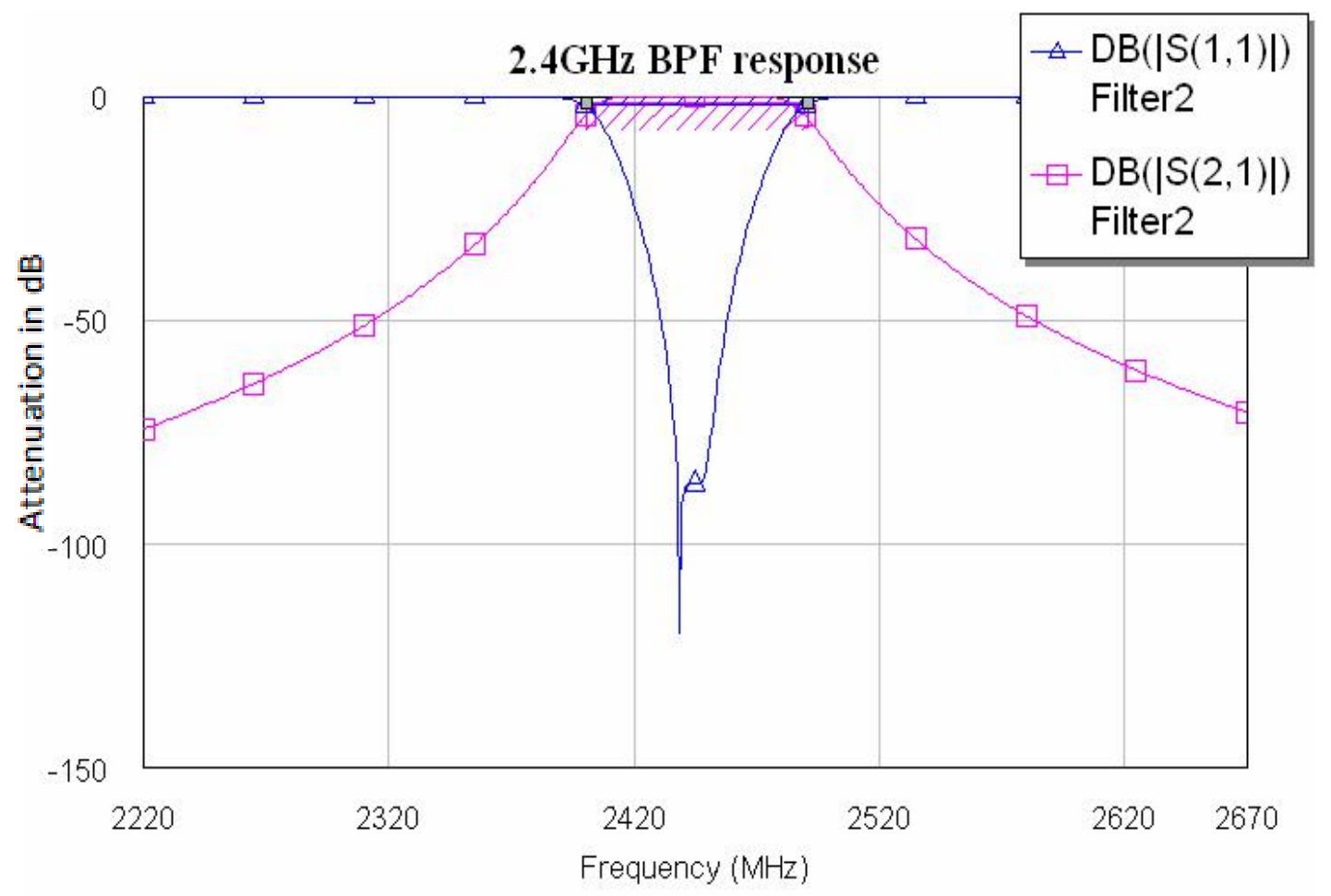

Figure no. 5: Response of $2.4 \mathrm{GHz}$ band pass filter

\section{2. $L N A$}

From the simulation of the LNA, it is seen that the transmission coefficient at the frequency of interest is $26.05 \mathrm{~dB}$ as shown in Figure no. 6. This gain is sufficient enough to minimize the noise contributed by the mixer which is quite high $(11.44 \mathrm{~dB})$. The NF of the LNA is found to be very low within the frequency band of interest as shown in Figure no. 7. This is very desirable since it will ensure that the overall noise of the receiver is very low. 


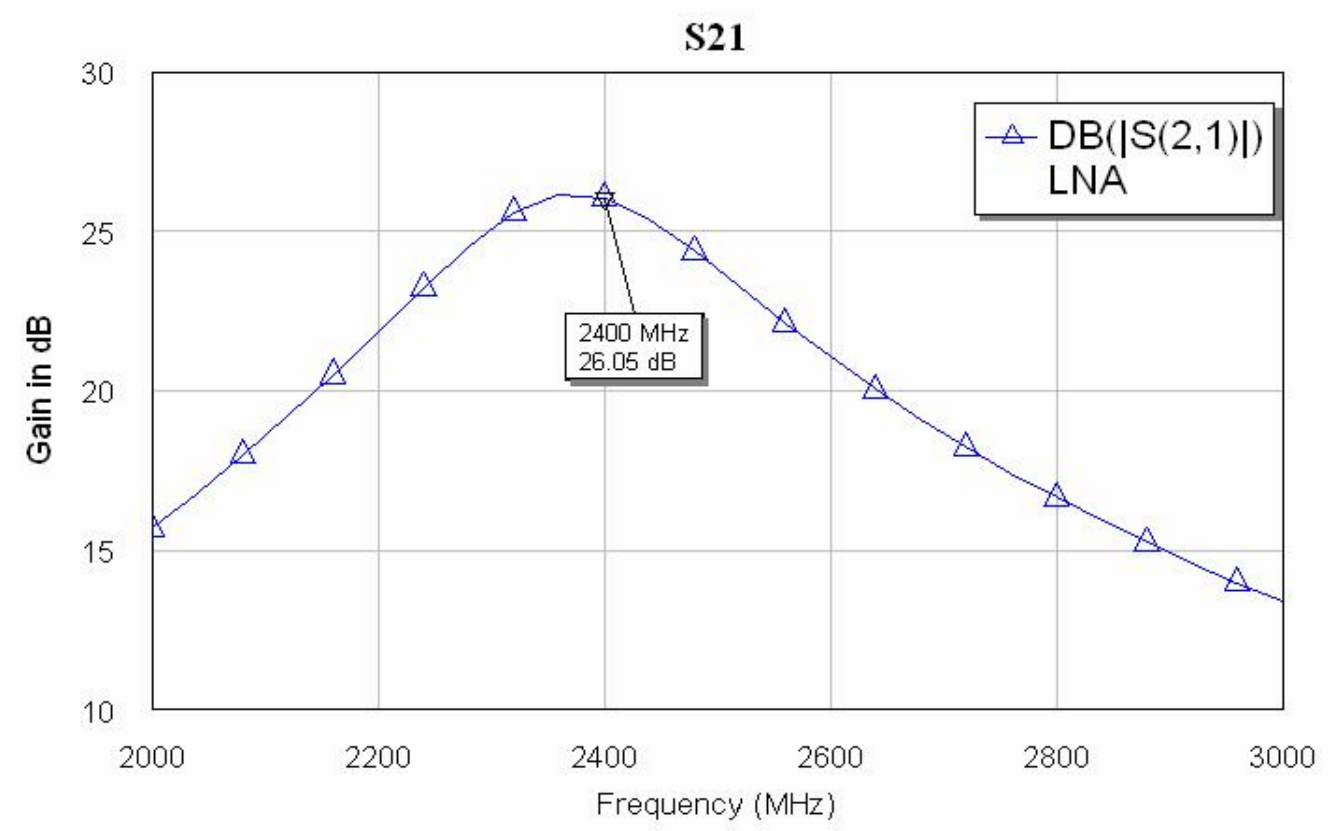

Figure no. 6: Gain of the LNA in $d B$

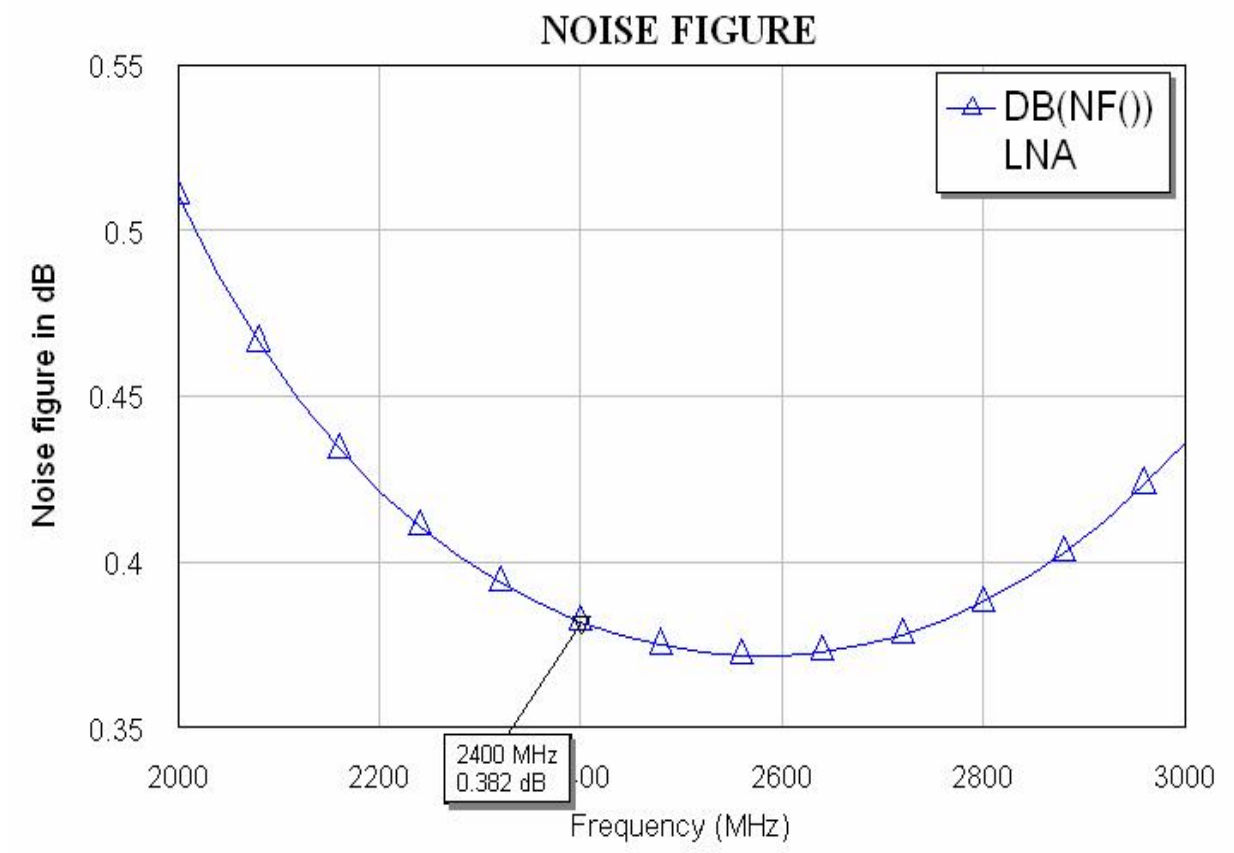

Figure no. 7: Noise figure of the LNA

\subsection{Image Filter}

The simulation result of the image filter is as shown in Figure no. 8. The filter has a $3 \mathrm{~dB}$ bandwidth of $80 \mathrm{MHz}$. An attenuation of $-18 \mathrm{~dB}$ is achieved at the $2.394 \mathrm{GHz}$ frequency. A higher order filter would have given a better attenuation but it would result in a higher value of both the input and output reflection coefficients $\left(\mathrm{S}_{11}\right.$ and $\mathrm{S}_{22}$ ) which is undesirable. Therefore a compromise has to be reached. 


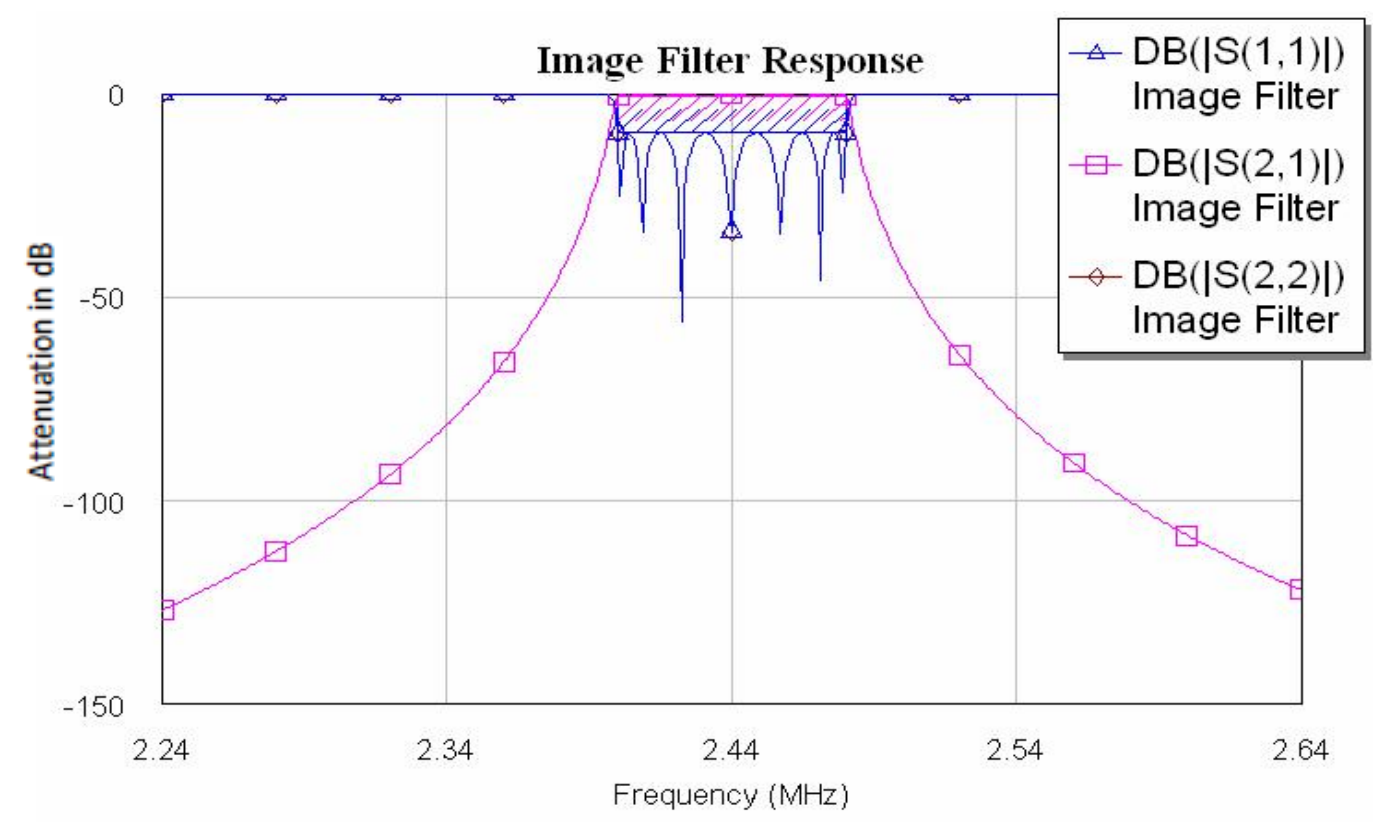

Figure no. 8: Response of the image filter

\subsection{IF Filter}

The IF filter is designed to have a return loss of $2 \mathrm{~dB}$. From the IF filter response in Figure no. 9, it is seen that both the reflection coefficient at the input $\left(\mathrm{S}_{11}\right)$ and the reflection coefficient at the output $\left(\mathrm{S}_{22}\right)$ are minimum around the center frequency of the filter which is a desirable property of the filter. It is also observed that the transmission coefficient is maximum within the frequency band of interest and an attenuation of $40 \mathrm{~dB}$ is attained at the 6.7 $\mathrm{MHz}$ and $14.7 \mathrm{~dB}$.

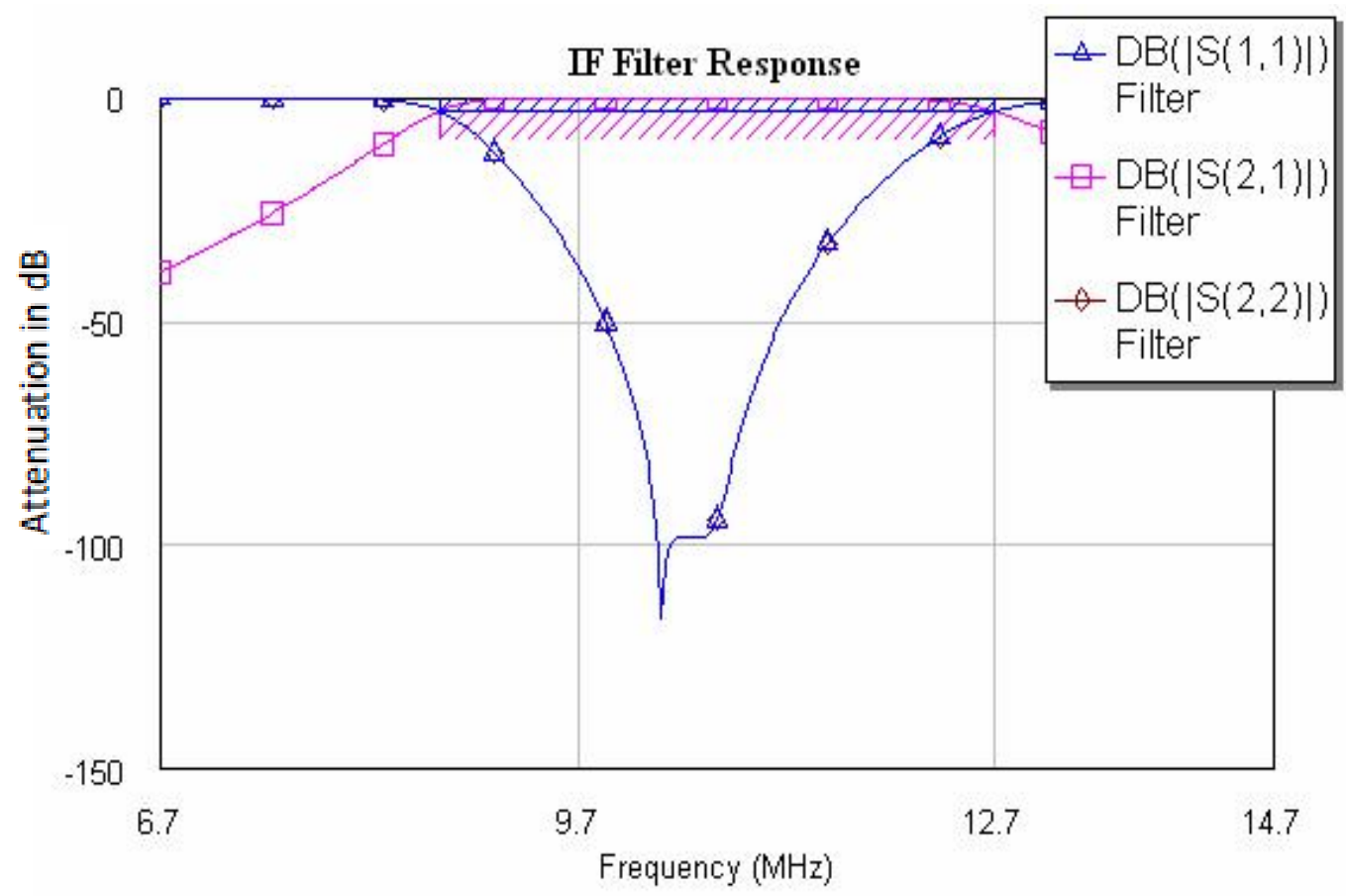

Figure no. 9: Frequency response of the IF filter 


\subsection{Receiver Path}

The entire receiver path is simulated using blocks on Microwave office environment as shown in Figure no. 10. The result of the simulation at the output of the IF filter is shown in Figure no 11. The IF signal has a frequency of $11 \mathrm{MHz}$ as expected. The output power of the receiver is determined to be $24 \mathrm{dBm}$ while the power at the input is $-13 \mathrm{dBm}$. This gives an overall gain of $10 \log \left(10^{25} \mathrm{~mW} / 0.05 \mathrm{~mW}\right)$ $=37 \mathrm{~dB}$ for the entire path. This gain is less than expected. This can be attributed to the finite losses in the filters since each filter contributes $3 \mathrm{~dB}$ attenuation at the bandpass. The mixer used has a negative conversion gain which implies that there is also some power loss at the mixer. As a result, the overall power gain is reduced.

The major noise contributors in the receiver path are the LNA and the mixer since the noise contributed by the IF amplifier is negligible. Therefore the total noise figure of the entire cascade is:

$$
\mathrm{NF}=0.382+(11.4 / 26.05)=0.821 \mathrm{~dB}
$$

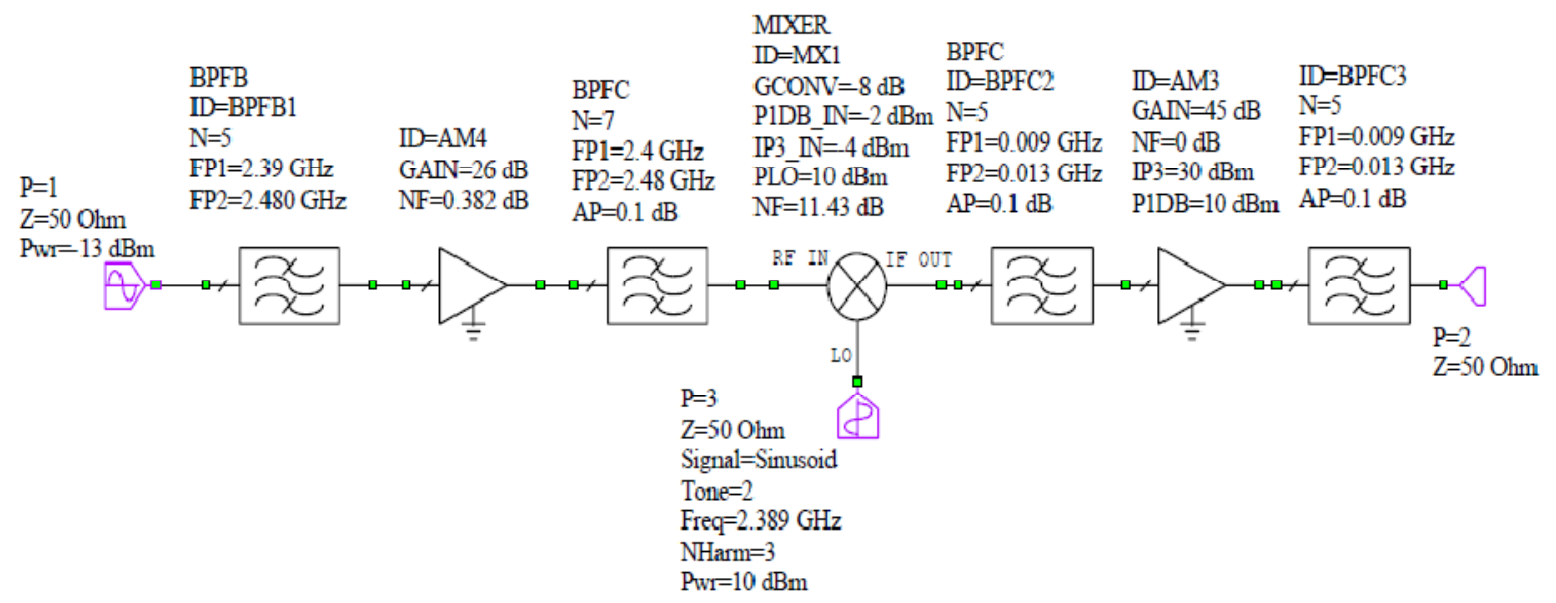

Figure no. 10: The entire receiver path

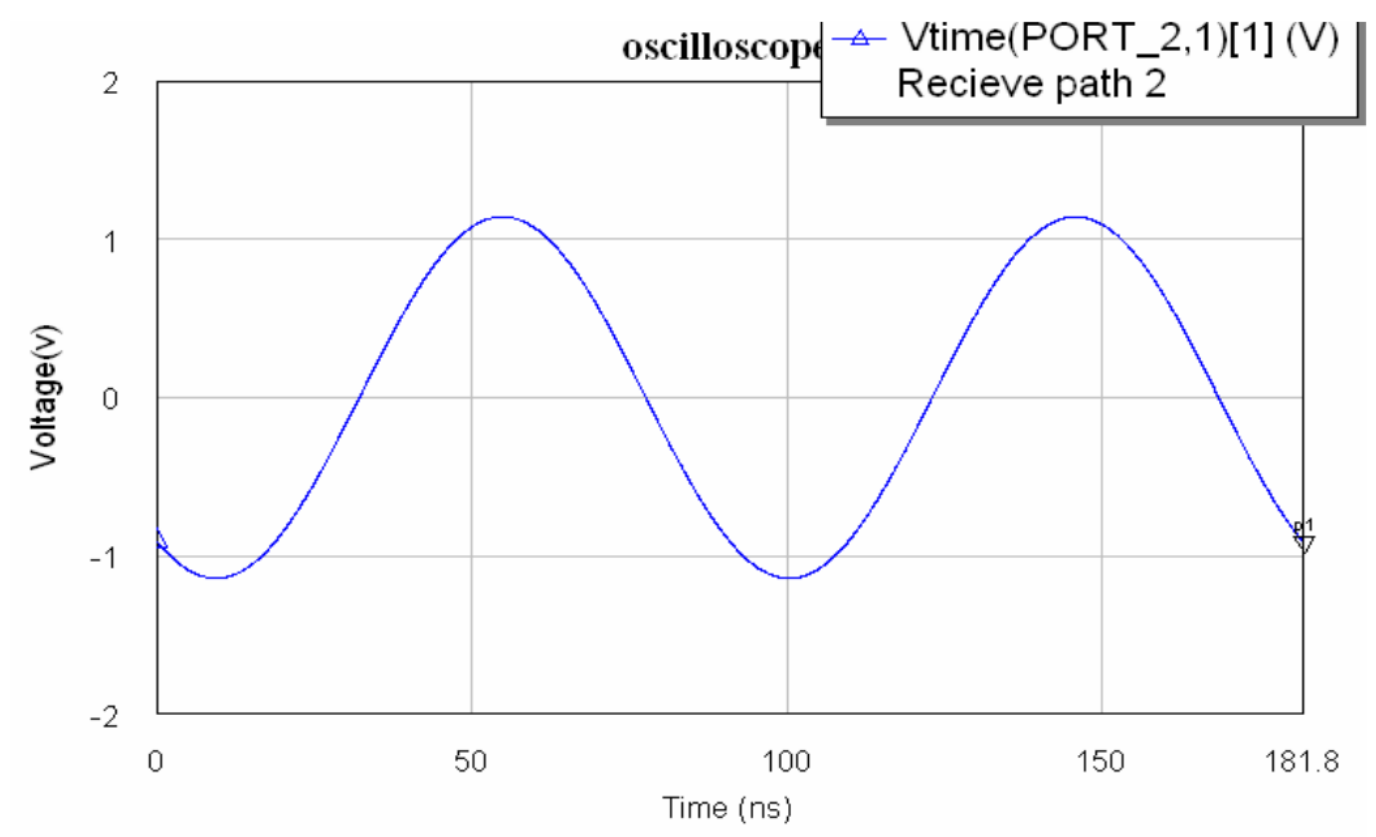

Figure no. 11: Waveform at the output of the IF filter in the receiver path 


\subsection{Transmitter Path}

The transmitter path is simulated as shown in Figure no. 12. The IF filter and the RF band-pass filter for both the transmitter and receiver have similar characteristics. Therefore the simulations given in sections 3.1 and 3.4 give adequate representation. The power amplifier gives an output of $19 \mathrm{dBm}$ for an input of $9 \mathrm{dBm}$. The output waveform of the transmitter path is shown in Figure no. 13. The signal has a frequency of $2.391 \mathrm{GHz}$.

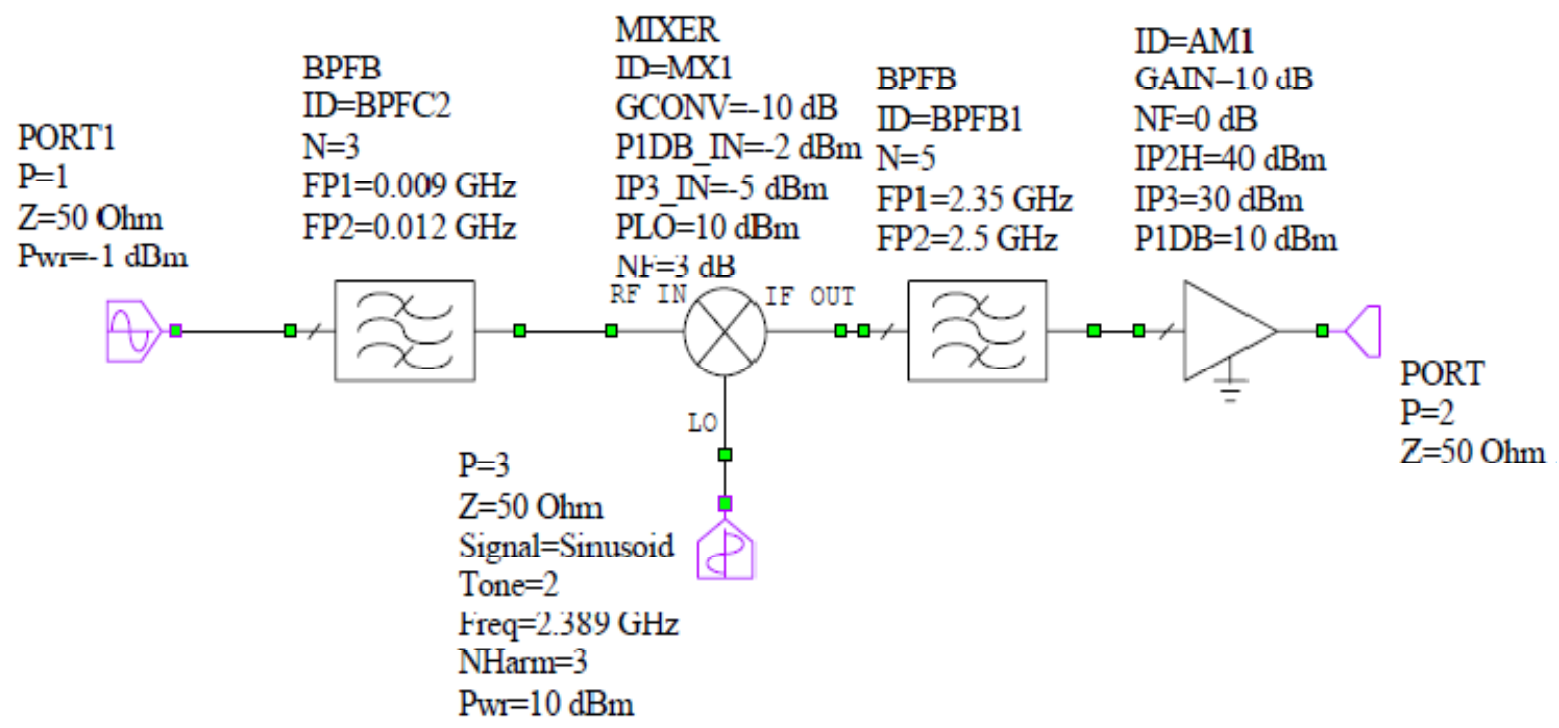

Figure no. 12: The transmitter path

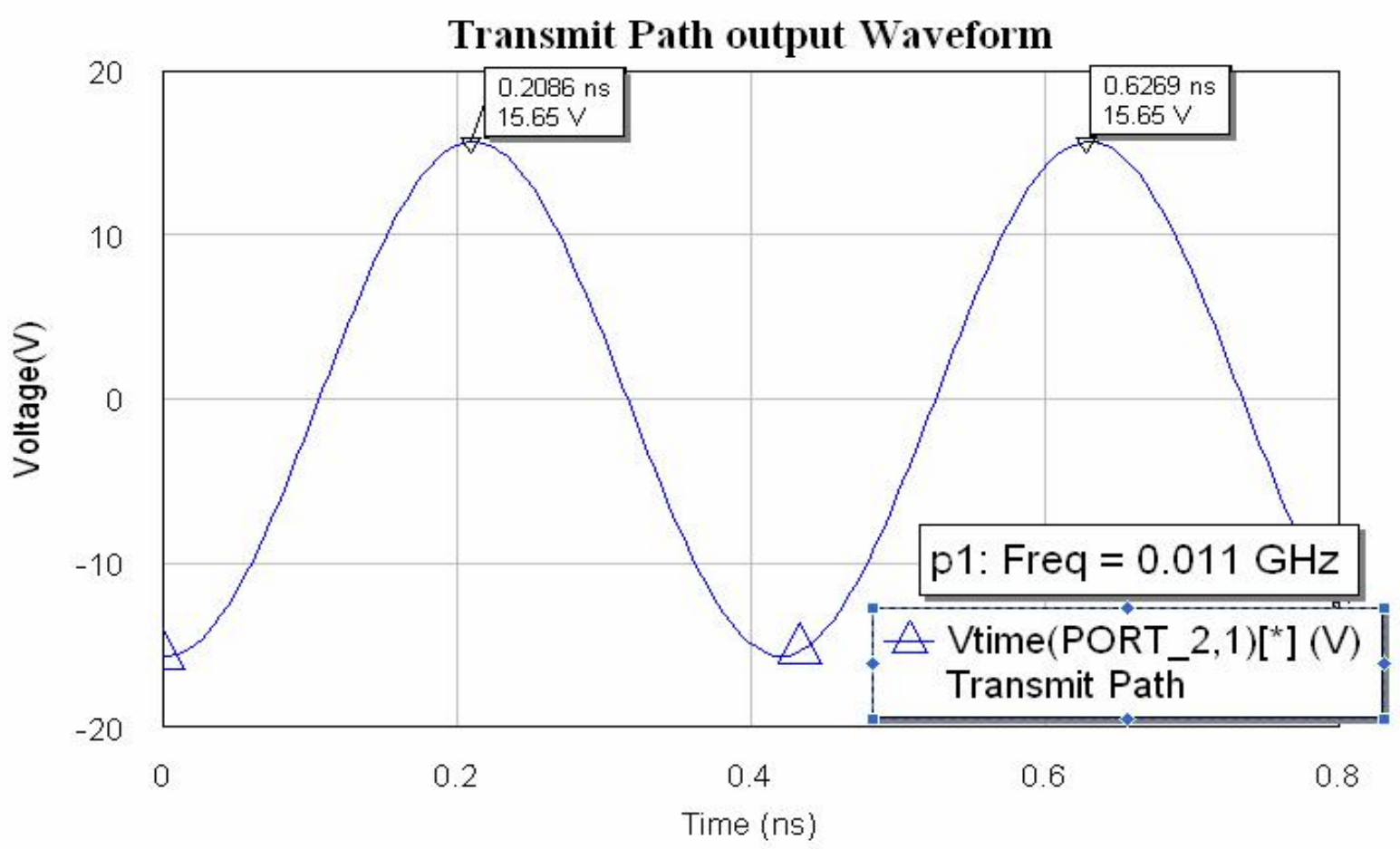

Figure no. 13: The output RF signal 


\section{Conclusion}

The functioning and the principle of operation of the heterodyne receiver have been successfully illustrated. The receiver has been covered in great detail and the simulations have been successfully done. However, the simulation of the power amplifier was not given and the power amplifier designed does not output large amounts of power. As a result, it is recommended that the power amplifier be designed to output more power of up to $1 \mathrm{~W}$. The actual implementation of the device could be done. However this may not be possible using discrete components, instead IC chips that have been designed for radio frequency design could be used for this implementation. Examples of such chips are RF2494 PCBA which comprise of the LNA, image filter and the down conversion mixer. The RF5117PCBA is a power amplifier chip. The RF2948PCBA comprise of the IF SAW filter, the IF amplifier, the up conversion mixer and the PA driver. The SI4136EVM is a dual PLL synthesizer that generates a frequency of $2.068 \mathrm{GHz}$ and $748 \mathrm{MHz}$. The RF2436PCBA is the transmitter/receiver switch. Other chips could also be used.

\section{REFERENCES}

Basavaraj, R. (2018). RF/microwave transceiver. Report, University of South Florida, USA. Camargo, E., \& Topacio, E. (1997). A $15 \mathrm{GHz}$ low cost wireless transceiver. IEEE MTT-S International Microwave and Optoelectronics Conference, Brazil.

Debski, W., Winkler, W., Genschow, D., \& Kraemer, R. (2011). 24 GHz transceiver front-end with integrated ramp generator. IEEE 6th European Microwave Integrated Circuit Conference, UK.

Rahayu, Y., Din, J., \& Abdulrahman, T. (2003). $23 \mathrm{GHz}$ transceiver system design for point-to-point microwave link. IEEE 4th National Conference of Telecommunication Technology, Malaysia. 